\title{
Empirical analysis of the Lieb-Oxford bound in ions and molecules
}

\author{
Mariana M. Odashima and K. Capelle \\ Instituto de Física de São Carlos \\ Universidade de São Paulo \\ Caixa Postal 369, São Carlos, 13560-970 SP, Brazil
}

November 1, 2018

\begin{abstract}
Universal properties of the Coulomb interaction energy apply to all many-electron systems. Bounds on the exchange-correlation energy, in particular, are important for the construction of improved density functionals. Here we investigate one such universal property - the LiebOxford lower bound - for ionic and molecular systems. In recent work [J. Chem. Phys. 127, 054106 (2007)], we observed that for atoms and electron liquids this bound may be substantially tightened. Calculations for a few ions and molecules suggested the same tendency, but were not conclusive due to the small number of systems considered. Here we extend that analysis to many different families of ions and molecules, and find that for these, too, the bound can be empirically tightened by a similar margin as for atoms and electron liquids. Tightening the Lieb-Oxford bound will have consequences for the performance of various approximate exchange-correlation functionals.
\end{abstract}

\section{Introduction}

The Lieb-Oxford lower bound [1] on the exchange-correlation $(x c)$ energy is an exact quantum-mechanical property of any Coulomb-interacting system. Originally, this bound was obtained in an investigation of a very fundamental problem of stability of matter 2]. An important practical application of the bound is as a constraint in the construction of approximate exchange-correlation functionals for density-functional theory (DFT).

In DFT [3, 4, 5, many-body effects on electronic structure are included by means of an approximate $x c$ functional. The Lieb-Oxford bound is one of the key ingredients in the construction of nonempirical $x c$ functionals [6], where it is usually written as

$$
E_{x c}[n] \geq-C \int d^{3} r n^{4 / 3}
$$


where $C$ is a positive universal constant whose exact value is unknown. A first estimate for the maximum value of this constant, $C_{L}=8.52$ [2] was later substituted [1] by the tighter value $C_{L O}=1.68$, which is adopted in most current work.

With this value of $C$, the bound is satisfied for instance by the local-density approximation (LDA) [7, 8], the generalized-gradient approximations (GGAs) PW91 9] and PBE [10, and the TPSS meta-GGA [11. On the other hand, earlier GGAs [12] and meta-GGAs [13] and other functionals containing fitting parameters [14, 15, 16, 17] are not guaranteed to satisfy the bound for all possible densities. The success of some these latter functionals shows that, while the bound is doubtlessly obeyed by the exact functional, satisfaction is not a necessary condition for good performance in practice.

In terms of the local-density approximation to the exchange energy,

$$
E_{x}^{L D A}[n]=-\frac{3}{4}\left(\frac{3}{\pi}\right)^{1 / 3} \int d^{3} r n^{4 / 3}
$$

Eq. (11) becomes

$$
E_{x c}[n] \geq \lambda E_{x}^{L D A}[n],
$$

where $\lambda=\frac{4}{3}\left(\frac{\pi}{3}\right)^{1 / 3} C$. The value corresponding to the Lieb-Oxford estimate $C_{L O}=1.68$ is $\lambda_{L O}=2.275$. The exact universal $\lambda$ and $C$ are unknown, but their existence is guaranteed by the Lieb-Oxford bound, and their values are constrained by $\lambda \leq \lambda_{L O}$ and $C \leq C_{L O}$, respectively.

More recently, Chan and Handy numerically obtained an additional small reduction of this upper limit of $C$ to $C_{C H}=1.6358$, implying $\lambda_{C H}=2.215$ [18. The present authors [19] empirically investigated the bound for atoms, model Hamiltonians and the electron liquid, as well as for a very restricted number of ions and molecules. It turned out that for all systems investigated there even the tightest previous formulation of the bound, employing $C_{C H}=$ 1.6358 , is still substantially too generous. Away from unphysical limiting cases of model Hamiltonians, $C_{\text {conjec }}=1.0$ was found to be appropriate for all stable real systems investigated there. In view of the impact a reduction of $C$ (or, equivalently, of $\lambda$ ) may have on current density functionals, this observation requires further investigation.

In the present paper, we enlarge the data base of the empirical investigation to include diverse families of ions and molecules, in order to accumulate further evidence and to be able to quantify the possible degree of tightening for molecular systems. To this end, we use high-precision Quantum Monte Carlo (QMC), configuration interaction (CI) and exact exchange (EXX) data to evaluate the ratio

$$
\frac{E_{x c}[n]}{E_{x}^{L D A}[n]}=: \lambda[n],
$$

for different families of ions and molecules, including first and second-row dimers, and various hydrides and oxides.

This paper is organized as follows: in Sec. 2 we discuss various ions from the $\mathrm{Be}, \mathrm{Li}, \mathrm{C}$ and $\mathrm{O}$ isoelectronic series, as well as monovalent cations, comparing 


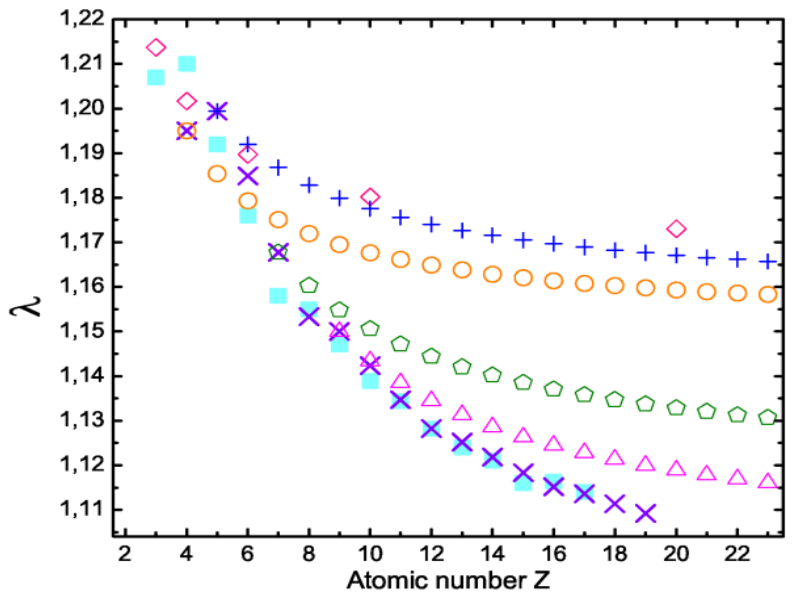

Figure 1: Value of the ratio $\lambda$, defined in Eq. (44), as a function of atomic number $Z$ for different ions. Ions isoelectronic to He (diamonds), Be (plus), Li (circles), $\mathrm{C}$ (pentagons) and $\mathrm{O}$ (triangles), and monovalent cations (crosses) are shown. Values for neutral atoms (squares) [19] are included for comparison.

their behaviour with data for the $H e$ isoelectronic series and atoms obtained in Ref. 19. Section 3 deals with different classes of molecules, and Sec. 4 provides a comparative analysis of the emerging trends. Section 5 contains our conclusions.

\section{Ions}

Results from evaluating $\lambda[n]$ for ions from the $\mathrm{He}, \mathrm{Be}, \mathrm{Li}, \mathrm{C}$ and $\mathrm{O}$ isoelectronic series, and for monovalent cations are displayed in Fig. 1 .

According to Eq.(44), in order to calculate the parameter $\lambda[n]$ we need the exact exchange-correlation energy $E_{x c}[n]$ and the LDA exchange energy $E_{x}^{L D A}[n]$. Ionic correlation energies were taken from Ref. [20, except for the He series, for which we employed the DMC $x c$ energy given by Huang and Umrigar 21. To these near-exact correlation energies we added EXX energies calculated with the OEP method [22, 23] to simulate the exact $x c$ energy. Small differences between exact exchange in DFT and in Hartree-Fock theory are negligible on the scale of effects we are after. The values for $E_{x}^{L D A}$ were obtained 23] from the LDA exchange functional and evaluated at self-consistent LDA(VWN) 8 densities.

Figure 1 1shows that ionic $\lambda$ values are similar to those of atoms, and far away from the theoretical upper limits. $\lambda[n]$ decreases as the ionic charge increases and the density distribution becomes more compact. Chemically similar ions (same number of electrons) fall on common $\lambda(Z)$ curves, displaying systematic trends as a function of atomic number and total charge. Monovalent cations have charge distributions that are very similar to neutral atoms, in particular 
for larger $Z$, and consequently have almost the same $\lambda$ values.

\section{Molecules}

Figure 2 reports $\lambda$ values for a set of dimers and hydrides. Figure 3 extends this comparison to oxides and a variety of other molecules for which near-exact correlation energies are available.

For the first-row dimers we employed highly-accurate diffusion Monte Carlo (DMC) correlation energies from Ref. 24] $\left(H_{2}\right)$ and Ref. 25] $\left(L i_{2}\right.$ to $\left.F_{2}\right)$. Correlation energies for the second-row dimers are from Ref. [26, where they were extracted from experimental dissociation energies, corrected for zero-point oscillations, by subtracting Hartree-Fock total energies. For the first-row hydrides we used DMC correlation energies extracted from Ref. [27, while correlation energies of second-row hydrides were obtained from experimental dissociation energies in Ref. [28]. For first and second-row oxides, as well as for the other molecules shown in Fig. 3, reference correlation energies are taken from 26] and 28 .

These correlation energies were combined with LDA exchange energies and Hartree-Fock exchange (again, small differences between exact exchange in DFT and in Hartree-Fock theory are negligible on the scale of effects we are after) to calculate the ratio $\lambda[n]=\left(E_{x}^{\text {exact }}+E_{c}^{\text {exact }}\right) / E_{x}^{L D A}$. The Hartree-Fock and LDA calculations were performed with the Gaussian 03 package 29]. These calculations used the $6-311 \mathrm{G}+(3 \mathrm{df}, 2 \mathrm{p})$ basis sets for the first-row dimers and hydrides, and the cc-pVTZ basis sets for the second-row dimers and hydrides, and all oxides and remaining molecules. Geometries for the Hartree-Fock and LDA calculations were taken from the references providing the correlation energies. For some molecules the geometries were not supplied together with the correlation energies [24. In these cases we performed our own geometry optimization on the MP2/cc-pVTZ level, followed by single-point calculations using the basis sets and methods indicated above.

Note that in Figs. 2 and 3 we display results as a function of the number of electrons $N$ of each molecule, instead of the atomic number $Z$ used for atoms and ions. Nevertheless, the tendencies observed for ionic atoms clearly persist for neutral molecules: $\lambda(N)$ decreases for larger (heavier) systems and increases for more rarefied electronic distributions; chemically similar families of systems fall on common $\lambda(N)$ curves; and values of $\lambda(N)$ are far from $\lambda_{L O}$.

The tendency of $\lambda$ to increase for more rarefied electronic distributions (lower average electron density) was already observed in the electron gas and for Hooke's atom [19. To further investigate this tendency for molecules, we also considered the variation of $\lambda$ as a function of the distance between the nuclei of the $H_{2}$ molecule. Approximate correlation energies of $H_{2}$ as a function of bond length are available from Ref. [30. From these data we find that for $\mathrm{H}_{2}$ at the equilibrium bond length $\lambda\left(R_{e}=1.401 a . u\right.$.) $=1.230$ (only slightly different from the value 1.251, obtained for the same system from DMC), whereas for stretched $H_{2}$ this changes to $\lambda\left(R_{e}=5.0 a . u.\right)=1.409$. Even this enhanced value 


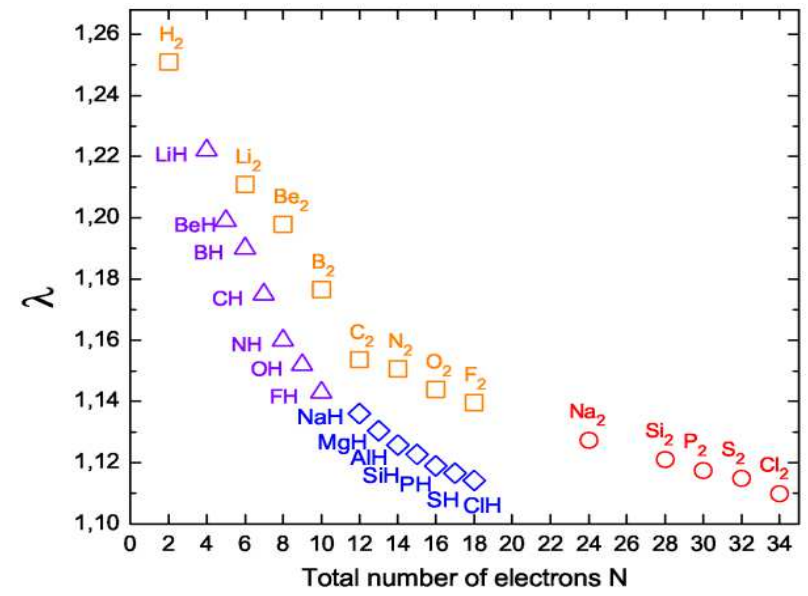

Figure 2: Energy ratio $\lambda$ as a function of the total number of electrons $N$, for first-row dimers (squares), second-row dimers (circles), first-row hydrides (triangles) and second-row hydrides (diamonds).

of $\lambda$, though, is still way below the maximum $\lambda_{L O}=2.275$.

\section{Analysis}

The $\lambda[n]$ functional, introduced in Ref. [19, has two key properties. One is that by definition it measures the inverse weight of LDA exchange relative to exact exchange and correlation: The larger $\lambda[n]$, the smaller is $\left|E_{x}^{L D A}[n]\right|$ relative to $\left|E_{x c}^{\text {exact }}[n]\right| . \lambda[n]$ therefore serves to characterize the importance of nonlocal exchange and of correlation in a given system or class of systems, indicating when $E_{x}^{L D A}[n]$ may be a good starting point and when more refined approaches are required.

The other key property is that the maximum of $\lambda[n]$ for a given class of systems provides a class-specific Lieb-Oxford bound. If these classes are large enough, such bounds may prove very useful in practice. Moreover, the maximum of $\lambda[n]$ across all investigated systems provides an empirical approximation to the universal constant $\lambda \leq \lambda_{L O}$ whose existence is guaranteed by the LiebOxford bound (3), but whose exact value remains unknown.

From the point of view of these two key properties, three common aspects of all $\lambda$ data presented in this paper deserve special attention:

(i) $\lambda$ decreases as the density distribution becomes more compact (e.g., for increasing $Z$ in isoelectronic series of ions). Larger $\lambda[n]$ arise for systems with spread-out rarefied density distribution. This trend is consistent with the characterization of very low-density systems as strongly correlated, which is rigorously correct for uniform electron liquids.

(ii) Chemically similar systems are characterized by common trends of $\lambda$, 


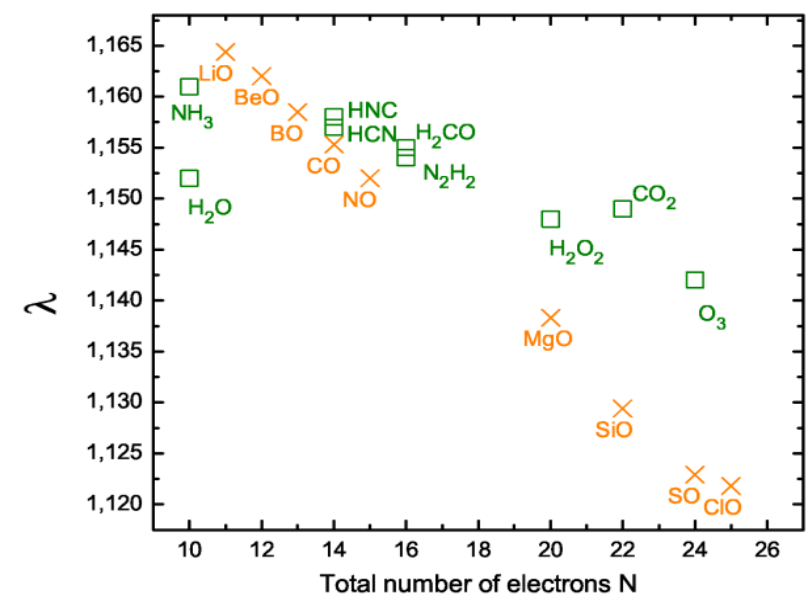

Figure 3: Energy ratio $\lambda$ as a function of the total number of electrons $N$, for first and second-row oxides (crosses) and a set of miscellaneous other molecules for which near-exact $E_{x c}$ data are available.

suggesting that $\lambda$ may be a useful parameter to characterize and classify systems, and that $\lambda[n]$ may be a useful ingredient in the construction of refined $x c$ functionals.

(iii) All values of $\lambda$ obtained are much smaller than the maximum value $\lambda_{L O}$, averaging at around half of $\lambda_{L O}=2.275$. The new ionic and molecular data reported here are fully consistent with the conclusion obtained in Ref. [19], and reinforce the conjecture that the Lieb-Oxford bound can be substantially tightened. The empirical value suggested in Ref. [19] for real systems (i.e. excluding unrealistic extreme limits of model Hamiltonians), $\lambda_{\text {conjec }} \approx 1.35$ (corresponding to $C_{\text {conjec }} \approx 1.0$ ) still covers all new systems investigated here, for which $\lambda$ never exceeds 1.26 .

If we include unstable systems, such as $H_{2}$ stretched to nearly four times its equilibrium distance, $\lambda[n]$ exceeds the value conjectured for stable systems at equilibrium (1.409 versus 1.35), which agrees with the trend observed for Hooke's atom in Ref. [19. Even at equilibrium, $H_{2}$ has with $\lambda=1.251$ the largest value found for any system investigated here, but this value is still clearly below the conjectured limit $\lambda_{\text {conjec }} \approx 1.35$.

\section{Conclusions}

Numerical results for the Lieb-Oxford parameter $\lambda[n]$, based on high-quality input data for the correlation energy $E_{c}[n]$, confirm the expectation of Ref. [19] that for all realistic systems the upper limit $\lambda_{L O}=2.275$ is too generous, by almost a factor of two. This is the same conclusion obtained in Ref. [19, but considerably strengthened by the present analysis covering many different types 
of ions and molecules not considered there.

Since we have not found a single exception to the emerging trend [31, our analysis suggests to pursue the following further investigations:

(i) Mathematical scrutiny of the Lieb-Oxford bound, which may not be the strongest possible bound for arbitrary Coulomb systems. Moreover, even if application to arbitrary systems (i.e. a truly universal bound) should require placing $\lambda$ at or near $\lambda_{L O}=2.275$, "realistic" systems, away from unphysical limits of system parameters, may allow a much smaller value of the upper limit. It remains to be clarified how to characterize such realistic systems or parameter values in a way independent of $\lambda$ itself.

(ii) Exploration of consequences a reduced value of $\lambda$ has for the perfor-

mance of common approximate density functionals. This exploration is aided by an investigation of the emerging systematic behaviour of $\lambda[n]$ accross different families of molecules, to obtain analytical or numerical models of the functional $\lambda[n]$.

Work on these issues is in progress in our group.

Acknowledgments This work was supported by FAPESP and CNPq.

\section{References}

[1] E. H. Lieb and S. Oxford, Int. J. Quantum Chem. 19, 427 (1981).

[2] E. H. Lieb, Phys. Lett. 70A, 444 (1979).

[3] W. Kohn, Rev. Mod. Phys. 71, 1253 (1999).

[4] R. M. Dreizler and E. K. U. Gross, Density Functional Theory (Springer, Berlin, 1990).

[5] R. G. Parr and W. Yang, Density-Functional Theory of Atoms and Molecules (Oxford University Press, Oxford, 1989).

[6] J. P. Perdew, A. Ruzsinszky, J. Tao, V. N. Staroverov, G. E. Scuseria and G. I. Csonka, J. Chem. Phys. 123, 062201 (2005).

[7] J. P. Perdew and Y. Wang, Phys. Rev. B 45, 13244 (1992).

[8] S. H. Vosko, L. Wilk and M. Nusair, Can. J. Phys. 58, 1200 (1980).

[9] J. P. Perdew, J. A. Chevary, S. H. Vosko, K. A. Jackson, M. R. Pederson, D. J. Singh and C. Fiolhais, Phys. Rev. B 46, 6671 (1992).

[10] J. P. Perdew, K. Burke and M. Ernzerhof, Phys. Rev. Lett. 77, 3865 (1996).

[11] J. Tao, J. P. Perdew, V. N. Staroverov and G. E. Scuseria, Phys. Rev. Lett. 91, 146401 (2003). 
[12] J. P. Perdew and Y. Wang, Phys. Rev. B 33, 8800 (1986). J. P. Perdew, Phys. Rev. B 33, 8822 (1986).

[13] J. P. Perdew, S. Kurth, A. Zupan and P. Blaha, Phys. Rev. Lett. 82, 2544 (1999).

[14] A. D. Becke, Phys. Rev. A 38, 3098 (1988).

[15] C. Lee, W. Yang and R. G. Parr, Phys. Rev. B 37, 785 (1988).

[16] Y. Zhang and W. Yang, Phys. Rev. Lett. 80, 890 (1998).

[17] A. D. Becke and E. R. Johnson, J. Chem. Phys. 127, 124108 (2007).

[18] G. K.-L.Chan and N. C. Handy, Phys. Rev. A 59, 3075 (1999).

[19] M. M. Odashima and K. Capelle, J. Chem. Phys. 127, 054106 (2007).

[20] E. R. Davidson, S. A. Hagstrom, S. J. Chakravorty, V. M. Umar, and C. Froese Fischer, Phys. Rev. A 44, 7071 (1991). S. J. Chakravorty, S. R. Gwaltney, E. R. Davidson, F. A. Parpia and C. Froese Fischer, Phys. Rev. A 47, 3649 (1993).

[21] C.-J. Huang and C. J. Umrigar, Phys. Rev. A 56, 290 (1997).

[22] J. B. Krieger, Y. Li and G. J. Iafrate, Phys. Rev. A 45, 101 (1992); ibid 46, 5453 (1992); ibid 47165 (1993).

[23] The self-consistent LDA and EXX-OEP calculations for atoms and ions reported here were performed with the mesh-based (basis-set free) atomic Kohn-Sham code written by E. Engel, University of Frankfurt, Germany.

[24] S. Manten and A. Lüchow, J. Chem. Phys. 115, 5362 (2001).

[25] C. Filippi and C. J. Umrigar, J. Chem. Phys. 105, 213 (1996).

[26] D. P. O'Neill and P. M. W. Gill, Molec. Phys. 103, 763 (2005).

[27] A. Lüchow and J. B. Anderson, J. Chem. Phys. 105, 7573 (1996).

[28] M. T. Carroll, R. F. W. Bader and S. H. Vosko, J. Phys. B 20, 3599 (1987).

[29] Gaussian 03, Revision B.04, M. J. Frisch et al., Gaussian, Inc., Pittsburgh PA, 2003.

[30] O. V. Gritsenko, P. R. T. Schipper and E. J. Baerends, J. Chem. Phys. 107, 5007 (1997).

[31] We conjecture that if there are any exceptions, i.e., systems with $\lambda[n] \approx$ $\lambda_{L O}$, these may be found in systems with dispersion interactions (e.g. van der Waals bonds), where the density distribution can become very rarefied. 